\title{
Mixed Connective Tissue Disease Associated with MPO-ANCA-positive Polyangiitis
}

\author{
Keisuke Kitaura, Tomoko Miyagawa, Kenichiro Asano, Sachiko Oouchi, \\ Takashi Miki, Takashi Fujisawa and Kenjiro Ishida
}

\begin{abstract}
The patient was a 42-year-old woman diagnosed as having MCTD and Sjögren's syndrome in 1989, and who was taking oral prednisolone. Proteinuria and microscopic hematuria were pointed out for the first time in December 2004. She was referred to our hospital because of massive hemoptysis. Advanced renal failure, anemia and pulmonary alveolar hemorrhage were diagnosed on admission. She was positive for serum MPOANCA. The patient was started on a therapy that included steroids, cyclophosphamide and plasmapheresis. However, her respiratory condition was untreatable and she died on the 16th day of hospitalization. The autopsy revealed alveolar hemorrhage in the lungs and crescentic glomerulonephritis. This patient was considered as a rare case of MCTD associated with MPO-ANCA-positive microscopic polyangiitis.
\end{abstract}

Key words: mixed connective tissue disease (MCTD), microscopic polyangiitis, pulmonary-renal syndrome, crescentic glomerulonephritis, MPO-ANCA

(DOI: 10.2169/internalmedicine.45.1703)

\section{Introduction}

Mixed connective tissue disease (MCTD) was first described by Sharp in 1972. It is characterized by a high titer of serum anti-U1-RNP antibody and mixed clinical features which are also present in systemic lupus erythematosus (SLE), systemic sclerosis (SSc), and polymyositis and dermatomyositis (PM/DM). Renal dysfunction occurs in 20$30 \%$ of the cases which present relatively mild abnormalities. As for glomerular lesions, membranous nephropathy is the most frequent pathology. Mesangial or membranoproliferative changes similar to lupus nephritis are occasionally mentioned (1-3). Characteristic findings in the scleroderma kidney - intimal thickening of small arteries and fibrinoid necrosis of arcuate and interlobular arteries - are observed as well. Acute loss of renal function caused by acute renal failure or crescentic glomerulonephritis has been rarely reported $(1,4)$. With regard to the lungs, pulmonary hypertension and/or fibrosis are generally described. However, just a few cases of alveolar hemorrhage have been reported $(5,6)$.

Microscopic polyangiitis (MPA) is an autoimmune disor- der in which inflammation of the small vessels (small arteries, capillaries and venules) affects mostly the lungs and kidneys. The antineutrophil cytoplasmic antibody which specifically binds to myeloperoxidase (MPO-ANCA) is detected in the serum with high frequency. Clinically, the kidney dysfunction could manifest as rapidly-progressive glomerulonephritis (RPGN). Histopathologically, necrotizing crescentic glomerulonephritis as well as inflammation of the small arteries are frequently observed. In the lungs, both interstitial pneumonia and alveolar hemorrhage have been reported. The lung disorder, especially alveolar hemorrhage is presumed to parallel the condition of the kidneys.

We recently encountered a case of MCTD which was associated with MPA. The pathological findings were closely examined at autopsy. This rare association of MCTD and MPO-ANCA-related MPA is discussed based on a review of previous reports. 


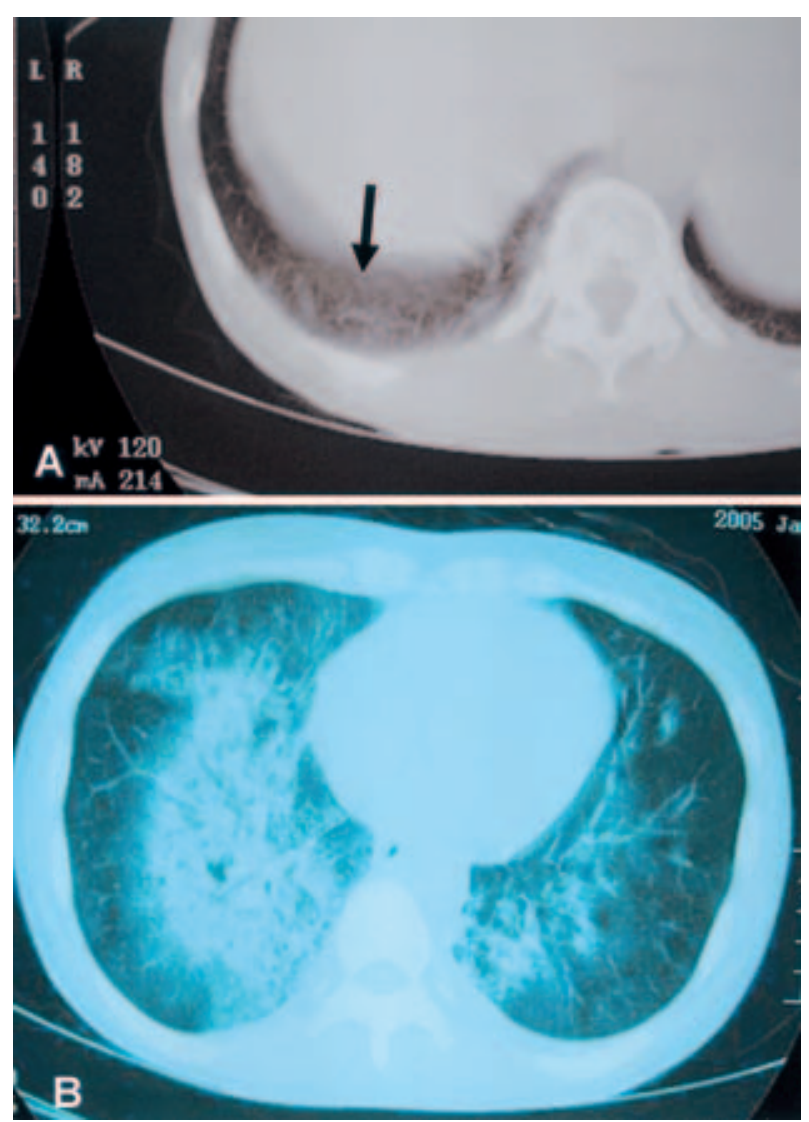

Figure 1. The chest CT obtained on admission shows fibrosis right underneath the visceral pleura in the posterior basal segment (arrow) (A), and alveolar hemorrhage around bilateral pulmonary hila which was more marked in the right lower lobe (B).

\section{Case Report}

\section{Clinical course}

A 42-year-old woman was referred to our hospital because of hemoptysis and dyspnea. She had been diagnosed as having MCTD and Sjögren's syndrome in 1989, and had been taking prednisolone $7.5 \mathrm{mg}$ per day for years. Low counts of blood leukocytes and platelets, and low concentrations of serum complements $(\mathrm{C} 3, \mathrm{C} 4)$ were recorded during the course. However, no pulmonary or renal function abnormalities had been observed. Abnormal urinary findings (proteinuria $1+$, microhematuria $3+$ ) were pointed out for the first time on December 9, 2004. Blood-stained sputa subsequently appeared on December 27. She finally suffered from massive hemoptysis and dyspnea on January 3, 2005 and was referred to our hospital for an emergency admission. Alveolar hemorrhage around bilateral pulmonary hila was prominent on the chest X-rays and CT (Fig. 1).

On admission, her blood pressure was $144 / 72 \mathrm{mmHg}$ and her body temperature was $37.6^{\circ} \mathrm{C}$. The physical examination revealed anemic conjunctivae, swollen fingers, sclerodactyly and slight edema in the lower limbs. Neither petechiae nor
Table 1. Laboratory Findings on Admission

\begin{tabular}{|c|c|c|c|c|c|}
\hline protein & + & $\mathrm{Na}$ & $144 \mathrm{mEq} / \mathrm{l}$ & ANA & $\times 2560$ \\
\hline occult blood & $3+$ & K & $5.4 \mathrm{mEg} / \mathrm{I}$ & pattern & hemogeneous \\
\hline \multirow[t]{2}{*}{$\mathrm{RBC}$} & $>100 / \mathrm{HF}$ & $\mathrm{Cl}$ & $116 \mathrm{mEq} / \mathrm{l}$ & Anti-ssDNA Ab & $14.5 \mathrm{IU} / \mathrm{I}$ \\
\hline & & $\mathrm{Ca}$ & $7.1 \mathrm{mg} / \mathrm{dl}$ & Anti-dsDNA Ab & $6.0 \mathrm{IU} / \mathrm{I}$ \\
\hline \multicolumn{2}{|c|}{ Arterial blood gas analysis } & $\mathrm{P}$ & $6.8 \mathrm{mg} / \mathrm{dl}$ & Anti-Sm Ab & negative \\
\hline $\mathrm{pH}$ & 7.318 & UA & $6.0 \mathrm{mg} / \mathrm{dl}$ & Anti-RNP Ab & $160 I U / I$ \\
\hline $\mathrm{PaCO} 2$ & $26.3 \mathrm{mmHg}$ & BUN & $94 \mathrm{mg} / \mathrm{dl}$ & Anti-SS-A Ab & $>500 \mathrm{U} / \mathrm{ml}$ \\
\hline $\mathrm{PaO} 2$ & $67.3 \mathrm{mmHg}$ & $\mathrm{Cr}$ & $6.0 \mathrm{mg} / \mathrm{dl}$ & Anti-SS-B Ab & $9.0 I U / I$ \\
\hline $\mathrm{HCO} 3$ & $15.8 \mathrm{mmol} / \mathrm{I}$ & TP & $5.1 \mathrm{~g} / \mathrm{dl}$ & Anti-Scl-70 Ab & negative \\
\hline \multirow[t]{3}{*}{$\mathrm{BE}$} & $-12.9 \mathrm{mmol} / \mathrm{I}$ & Alb & $2.0 \mathrm{~g} / \mathrm{dl}$ & MPO-ANCA & $132 \mathrm{EU}$ \\
\hline & & T-Bil & $0.3 \mathrm{mg} / \mathrm{dl}$ & PR3-ANCA & negative \\
\hline & & AST & $119 \mathrm{IU} / \mathrm{I}$ & Anti-GBM Ab & negative \\
\hline Peripheral blood & & ALT & $54 I U / I$ & $\mathrm{C} 3$ & $36 \mathrm{mg} / \mathrm{dl}$ \\
\hline WBC & $5500 / \mu 1$ & LDH & 979IU/I & $\mathrm{C} 4$ & $8 \mathrm{mg} / \mathrm{dl}$ \\
\hline RBC & $178 \times 10^{4} / \mu 1$ & ALP & $1109 \mathrm{IU} / \mathrm{I}$ & $\mathrm{CH} 50$ & $<10 \mathrm{U} / \mathrm{ml}$ \\
\hline $\mathrm{Hb}$ & $4.9 \mathrm{~g} / \mathrm{dl}$ & $\gamma$-GTP & $287 \mathrm{IU} / \mathrm{I}$ & CRP & $6.8 \mathrm{mg} / \mathrm{dl}$ \\
\hline $\mathrm{Ht}$ & $15.4 \%$ & AMY & $258 \mathrm{IU} / \mathrm{I}$ & $\mathrm{HBs} \mathrm{Ag}$ & negative \\
\hline PLT & $6.1 \times 10^{4} / \mu 1$ & Glu & $96 \mathrm{mg} / \mathrm{dl}$ & HCV $A b$ & negative \\
\hline
\end{tabular}

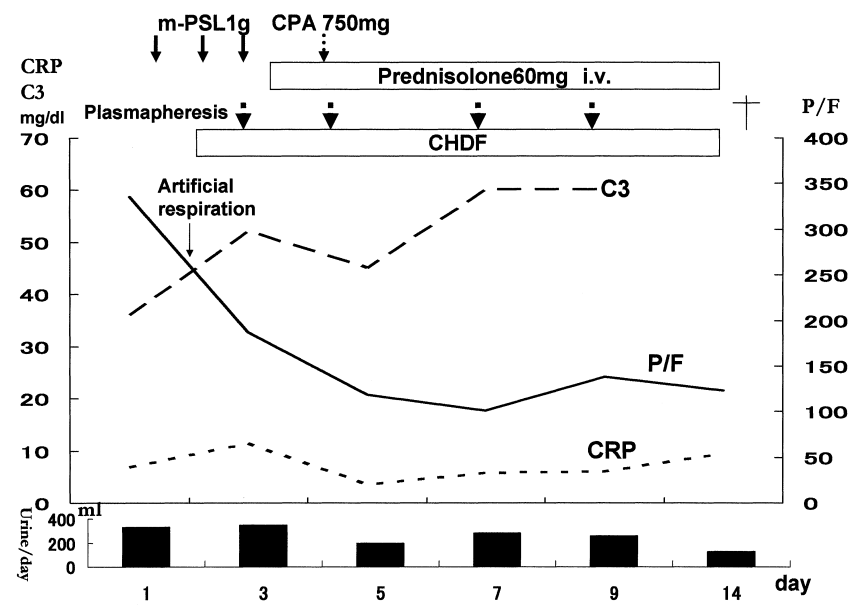

Figure 2. Clinical course. $\mathrm{P} / \mathrm{F}: \mathrm{PaO}_{2} / \mathrm{FiO}_{2}$ ratio.

subcutaneous bleeding was observed on the skin. Coarse crackle sounds were audible in all lung fields. Laboratory findings are shown in Table 1. Advanced renal failure with microhematuria and proteinuria, severe anemia, thrombocytopenia and elevated C-reactive protein were noted. An arterial blood gas sampling showed hypoxemia and metabolic acidosis. Serum C3, C4 concentrations and CH50 (50\% hemolytic unit of complement) were remarkably low. One week after admission, her serum tested positive for antinuclear antibody, anti-RNP antibody, anti-SS-A antibody and MPO-ANCA with high titers, and negative for anti-dsDNA antibody, anti-Sm antibody and anti-GBM antibody.

Her clinical course is shown in Fig. 2. The onset with abnormal urinary findings and the successive acute worsening of renal failure were compatible with RPGN. In addition, she had hemoptysis and a profuse alveolar hemorrhage as shown on the chest CT. She presented with pulmonary-renal syndrome on admission, although the origin of the disease was to be specified. Since prompt reduction of vasculitis was critical, a steroid pulse therapy with $1 \mathrm{~g}$ of methylprednisolone per day was started immediately. Artificial respiratory support and a continuous hemodiafiltration (CHDF) were attempted on the second day. The marked hypocomple- 


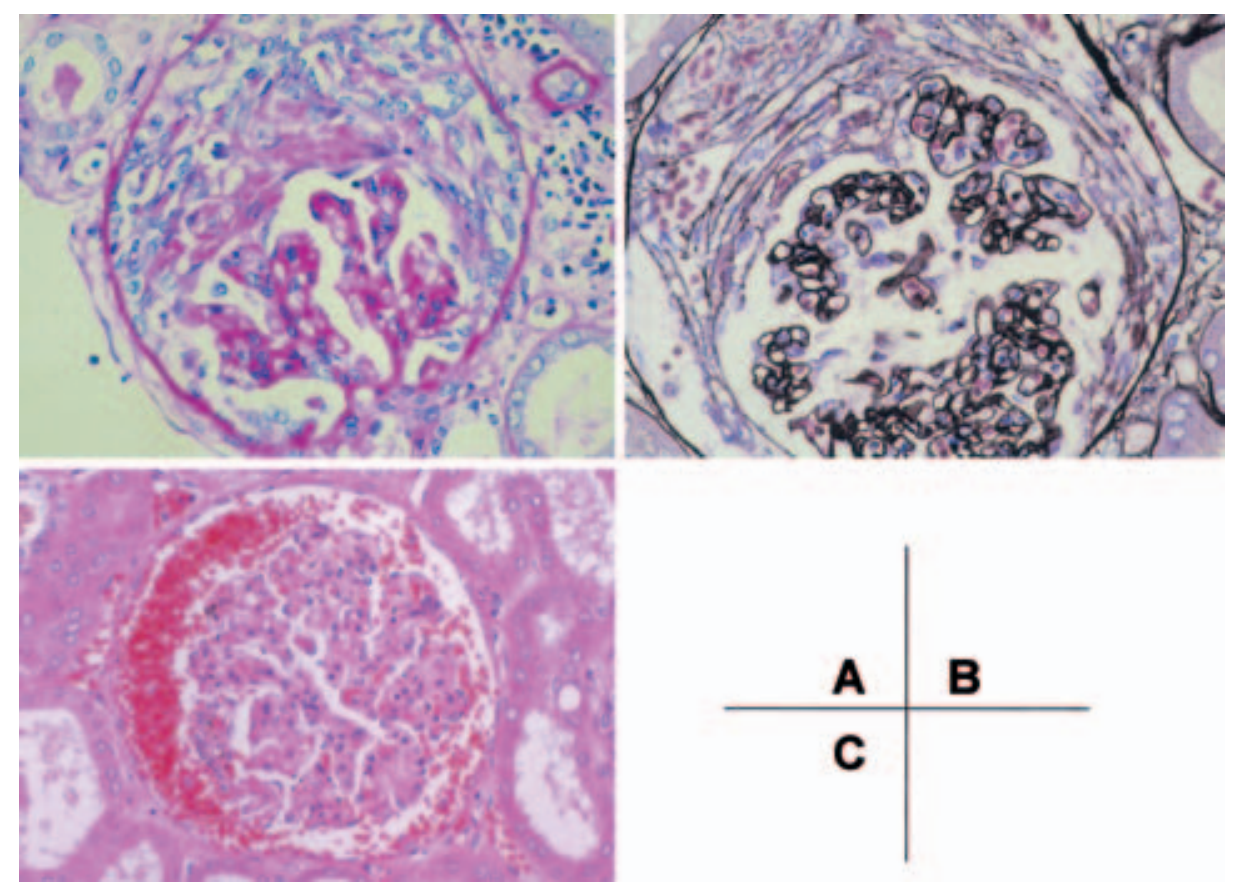

Figure 3. Light microscopic appearance of a glomerulus with a cellular crescent and contracted glomerular tuft (A), without glomerular basement membranous change (B), red blood cells in Bowman's capsule $($ C), A: Periodic acid Schiff hematoxylin (PAS) $\times \mathbf{4 0 0}$, B: Periodic acid silver methenamine $(\mathrm{PAM}) \times 400$, C: HE stain $\times 400$.
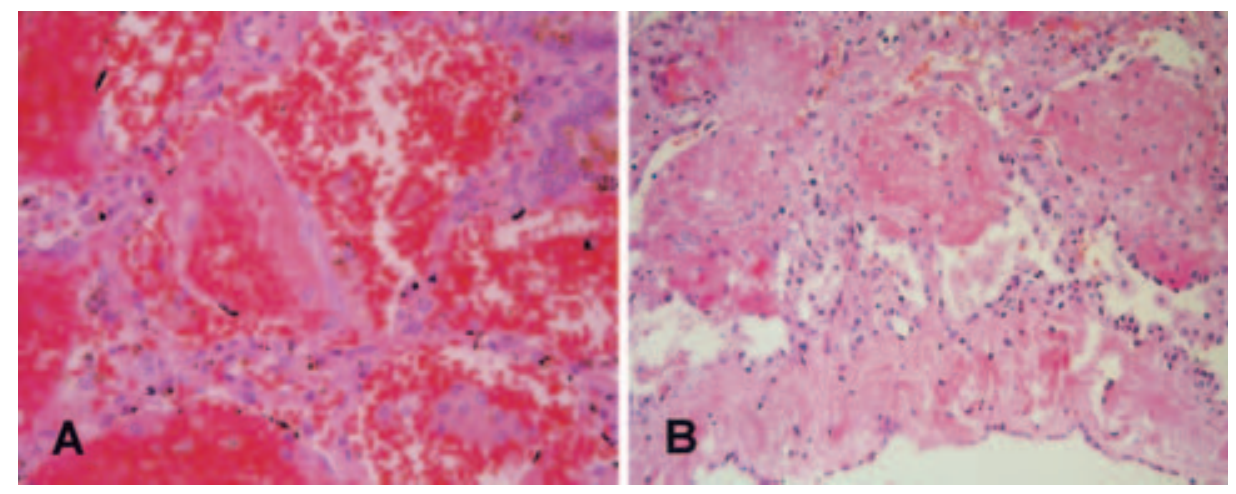

Figure 4. A massive number of red blood cells are observed in the alveolar space (A); fibrosis was found underneath the visceral pleura (B) A, B: HE stain $\times 400$.

mentemia was thought to represent systemic deposition of immune complex as observed in SLE. Therefore, plasmapheresis with fresh frozen plasma and intravenous cyclophosphamide pulse therapy were added. After 4 sessions of plasmapheresis, the serum complement concentration increased. However, respiratory failure secondary to alveolar hemorrhage persisted. The patient died on the 16th day of hospitalization.

\section{Autopsy findings}

An autopsy was performed with the consent of her family. The kidneys were slightly increased in size without ureteral obstruction. Microscopically, 75 glomeruli were evaluated in a cut specimen (Fig. 3A, B, C). Crescents were observed around $53 \%$ of the examined glomeruli (cellular crescents:
35/75, fibrous crescents: 5/75). Red blood cells were observed in the Bowman's capsules, which suggested minute bleeding caused by acute injury of glomerular capillaries. No glomerulosclerosis, mesangial proliferation or subendothelial deposits were observed. No renal tubular atrophy, interstitial fibrosis, lymphocyte infiltration or vasculitis of the interlobular arteries were found, either. Fibrinoid necrosis of arterioles and onion skin-like intimal thicking of small arteries were not found. Immunostaining of the glomeruli tested negative for immunoglobulins and complements. In the lungs, marked alveolar hemorrhage was found (Fig. 4A). In addition, fibrotic changes were observed in bilateral lower lobes, right underneath the visceral pleura (Fig. 4B). Systemically, no obvious inflammation of arteries was observed at the autopsy, which indicated that vascular injury would be 
Table 2. Findings of the Reported Case Which were Consistent with the Diagnostic Criteria of MCTD, Established by the Committee on Collagen Diseases, from the Ministry of Health, Labour and Welfare of Japan in 1996

\begin{tabular}{llll} 
& Common & SLE -like & SSc-like \\
\hline Physical findings & swollen fingers & & sclerodactyly \\
& & & \\
& & & \\
Serological findings fibrosis & ANA (+) & leukocytopenia & \\
& anti-RNP Ab (+) & thrombocytopenia & \\
\hline
\end{tabular}

confined to capillaries of the lungs and kidneys in this patient. In the heart, no pathological change was found in cardiac valves and ventricular walls. Inflammatory changes in the liver, pancreas and thyroid tissue, which occasionally develop in Sjögren's syndrome, were not observed. No malignant change was found in the lymph nodes and bone marrow. No specific findings were detected in other organs.

\section{Discussion}

This is a case report of pulmonary-renal syndrome which occurred in an MCTD patient who had been taking oral prednisolone for 15 years without any symptom of renal dysfunction. The diagnosis of MCTD had been made previously because the clinical findings fulfilled generallyaccepted diagnostic criteria of MCTD (Table 2). Namely, anti-RNP antibody was positive with a high titer, and clinical features which resembled those of SLE and scleroderma were shown (pancytopenia, sclerodactyly, and lung fibrosis). Although careful consideration for differential diagnosis was required, SSc was rather unlikely because anti-Scl-70 antibody was negative and hypocomplementemia was present. Although some clinical features which are characteristic to distinct autoimmune disorders could co-exist in an MCTD patient, pulmonary-renal syndrome is uncommon. Therefore, an overlapping immunological disorder such as MPA or Goodpasture's syndrome was suggested. Moreover, the marked decrease of serum complement concentrations implicated aggravation of an SLE-like condition in which immune complexes were actively formed. In addition to the possibility of overlapping MPA, it was also deducible that acute exacerbation of SLE-like vasculitis could have affected the lungs and the kidneys. Whatever the reason, the serious condition of the patient on admission required a rapid treatment of the disease which included steroid pulse therapy with $1 \mathrm{~g}$ of intravenous methylprednisolone for 3 days followed by $60 \mathrm{mg}$ of prednisolone, cyclophosphamide pulse therapy and plasmapheresis. During the therapy, the result of MPO-ANCA highlighted the possibility that overlapping MPO-ANCA-associated polyangiitis was the cause of the acute pulmonary and renal disorders. Goodpasture's syn-

Table 3. Diseases Associated with High Titers of Serum ANCA

MPO-ANCA

\author{
Microscopic polyangiitis (MPA) \\ Allergic granulomatous angiitis \\ (Churg-Strauss syndrome) \\ Necrotizing crescentic glomerulonephritis \\ Systemic lupus erythematosus (SLE) \\ Ulcerative colitis \\ Primary sclerosing cholangitis
}

drome was denied once her serum tested negative for antiGBM antibody. Despite these intensive therapies, the patient died on the 16th day of hospitalization because the respiratory failure was untreatable.

The histopathological examination of the kidneys at the autopsy showed $50 \%$ of the glomeruli presented cellular crescents. This finding supported the notion that overlapping MPA was the predominant cause of renal failure. Focal bleeding into the Bowman's capsules as observed in this case was additional evidence of acute injury of glomerular capillaries. No direct evidence of vasculitis - inflammation of the small arteries- was clearly demonstrated. However, based on previous observations, crescentic glomerulonephritis is presently explained to be clinically identical to MPA if the serum is positive for MPO-ANCA. Glomerular lesions are reported in approximately $20 \%$ of MCTD patients. The histology mostly resembled membranous nephropathy or mesangio-capillary proliferation as frequently observed in lupus nephritis (1-3). In this case, however, no mesangial proliferation or glomerular deposits were observed. Although the therapeutic effects of the steroids and cyclophosphamide were not negligible, this observation strongly suggested that the initial cause of glomerulonephritis differed from deposition of immune complexes. In addition, glomerulosclerosis and interstitial changes, which usually imply chronic glomerular injury, were absent. All these findings as mentioned above indicated that MCTD had exerted minimal effects on the glomeruli before the onset of pulmonary-renal syndrome in this patient.

MPO-ANCA could be found in various diseases as listed in Table 3. It is not specific for ANCA-related glomerulonephritis. However, Jennette et al reported that the positive predictor value of ANCA-related glomerulonephritis was as high as $98 \%$ when the MPO-ANCA was positive in RPGN (7). In contrast, Gross et al examined 32 MCTD patients, but no ANCA-positive cases were detected (8). From these results, the reported case was considered to be a rare example of MCTD associated with MPO-ANCA-related MPA. There are a few previous reports which referred to similar conditions. Interestingly, Germain and Davidman and Leatherman et al described a pulmonary-renal syndrome which 
occurred in MCTD patients, although their patients were examined before serum ANCA determination came into practice $(5,6)$. Li et al reported two cases of MCTD in which serum MPO-ANCA turned positive (9). The titers were 52 $\mathrm{U} / \mathrm{ml}$ and $80 \mathrm{U} / \mathrm{ml}$, respectively. A renal biopsy was performed in one case, and histopathologically diffuse glomerulonephritis was detected, which was consistent with the diagnosis of lupus nephritis, type IV according to the WHO morphologic classification. Makita et al reported an MCTD patient with a clinical picture that resembled that of our patient (10). The titer of MPO-ANCA was $118 \mathrm{U} / \mathrm{ml}$ and the kidneys presented crescentic glomerulonephritis (cellular crescents 5/63, fibrous crescents 10/63). Mild staining of IgM was shown in the mesangial region by immunofluorescence techniques. Tomioka et al reported ANCA-related crescentic glomerulonephritis in a patient with scleroderma (11). Previous reports on association of SSc and MPOANCA should be carefully considered since ANCA-related glomerulonephritis is presently recognized as a second form of renal crisis in SSc. The relatively high incidence of MPO-ANCA-related diseases in East Asia as described herein is assumed to be linked to variation of HLA haplotype.

As to the SLE-like aspect of MCTD, the marked hypocomplementemia detected on admission suggested that accelerated immune complex formation correlated with the acute loss of renal function. However, this possibility was denied by the autopsy since no glomerular deposit was found, despite the 16 days of intensive therapies. Kitridou et al reported that the decrement of $\mathrm{CH} 50$ and the incidence of nephropathy did not correlate in MCTD (1). The significance of decreased complements found in the evaluation of MCTD activity remains to be clarified. From anemia and thrombocytopenia on admission, thrombotic thrombocytopenic purpura (TTP) and hemophagocytic syndrome should be considered as possible complications in this case. However, neither intravascular thrombi nor hemophagocytosis was found in the autopsy specimens. Tubulointerstitial nephritis which is characteristic of Sjögren's syndrome was not apparent at the autopsy. Although a diagnosis of Sjögren's syndrome was recorded previously, re-evaluation of sicca syndrome was impossible on admission because of the critical condition of the patient.

Two pathological findings were pointed out in the lungs, i.e. massive alveolar hemorrhage and fibrosis right underneath the visceral pleura in the lower lobes. In the case described by Makita et al mild interstitial changes were observed in the lower lung fields as demonstrated by CT (10). This was recognized as SSc-like manifestation of MCTD because the lung fibrosis had been detected as early as 10 years before the onset of MPO-ANCA-associated glomerulonephritis. In MPO-ANCA-related MPA, pathological changes in the lungs usually develop in accordance with rapid deterioration of the renal function (12). Therefore, it is probable that in this case alveolar hemorrhage occurred due to overlapping MPA, and that fibrosis appeared as a SSclike change in MCTD. Respiratory failure is the main reason for mortality in pulmonary-renal syndrome because the impaired renal function is totally replaceable by using blood purification techniques. In the present patient, alveolar hemorrhage had progressed to such an extent that it was untreatable even under artificial respiratory support. The appearance of hemosputa one week before the massive hemoptysis suggests the possibility that an earlier diagnosis of pulmonary-renal syndrome could have resulted in a better outcome for this patient. Determination of serum ANCA may help perceive the onset of vasculitis promptly in order to carry out appropriate therapies timely.

\section{Conclusion}

A rare case of MCTD which was associated with MPOANCA-related MPA is described. Pulmonary-renal syndrome which is caused by MPA should be considered as a possible complication in MCTD patients. Determination of ANCA in MCTD patients may lead to a prompt diagnosis of pulmonary-renal syndrome, which might bring about a better outcome for patients.

\section{References}

1. Kitridou RC, Akmal M, Turkel SB, Ehresmann GR, Quismorio FP Jr, Massry SG. Renal involvement in mixed connnective tissue disease: a longitudinal clinicopathologic study. Semin Arthritis Rheum 16: 135-145, 1986.

2. Kobayashi S, Nagase M, Kimura M, Ohyama K, Ikeya M, Honda $\mathrm{N}$. Renal involvement in mixed connnective tissue disease. Report of 5 cases. Am J Nephrol 5: 282-289, 1985.

3. Sawai T, Murakami K, Kurasono T. Morphometric analysis of the kidney lesions in mixed connnective tissue disease (MCTD). Tohoku J Exp Med 174: 141-154, 1994.

4. Jones MB, Osterholm RK, Wilson RB, Martin FH, Commers JR, Bachmayer JD. Fatal pulmonary hypertension and resolving immune-complex glomerulonephritis in mixed connnective tissue disease. Am J Med 65: 855-863, 1978.

5. Germain MJ, Davidman M. Pulmonary hemorrhage and acute renal failure in a patient with mixed connnective tissue disease. Am
J Kidney Dis 3: 420-424, 1984.

6. Leatherman JW, Sibley RK, Davies SF. Diffuse intrapulmonary hemorrhage and glomerulonephritis unrelated to anti-glomerular basement membrane antibody. Am J Med 72: 401-410, 1982.

7. Jennette JC, Wilkman AS, Falk RJ. Diagnostic predictive value of ANCA serology. Kidney Int 53: 796-798, 1998.

8. Gross WL, Schmitt WH, Csernok E. Antineutrophil cytoplasmic autoantibody-associated diseases: a rheumatologist's perspective. Am J Kidney Dis 18: 175-179, 1991.

9. Li PK, Leung JC, Lai FM. Use of antineutrophil cytoplasmic autoantibodies in diagnosing vasculitis in a Chinese patient population. Am J Nephrol 14: 99-105, 1994.

10. Makita N, Katori H, Takemoto F, et al. A case of mixed connnective tissue disease (MCTD) complicated with MPO-ANCA-related necrotizing glomerulonephritis. Clin Nephro 154: 164-168, 2000.

11. Tomioka M, Hinoshita F, Miyauchi N, Akiyama Y, Saima S, Hiroe 
DOI: $10.2169 /$ internalmedicine.45.1703

M. ANCA-related crescentic glomerulonephritis in a patient with scleroderma without marked dermatological change and malignant hypertension. Intern Med 43: 496-502, 2004.

12. Arimura $Y$, Minoshima S, Tanaka U, et al. Pulmonary involvement in patients with myeloperoxidase specific-antineutrophil cytoplasmic antibody. Ryumachi 35: 46-55, 1995 (in Japanese with English Abstract).

(C) 2006 The Japanese Society of Internal Medicine http://www.naika.or.jp/imindex.html 